\title{
Multi-Angle Deformation Analysis of Hoffa's Fat Pad
}

\author{
Kevin Stevenson ${ }^{a}$, Mark Schweitzer ${ }^{b}$, Ghassan Hamarneh ${ }^{a *}$ \\ ${ }^{a}$ School of Computing Science, Simon Fraser University, Burnaby, BC, V5A 1S6, Canada \\ ${ }^{b}$ Department of Radiology and Orthopedic Surgery, NYU School of Medicine, Hospital for \\ Joint Diseases, New York, NY 10016, USA
}

\begin{abstract}
Recent advances in medical research hypothesize that certain body fat, in addition to having a classical role of energy storage, may also have mechanical function. In particular, we analyzed the infrapatellar fat pad of Hoffa using 3D CT images of the knee at multiple angles to determine how the fat pad changes shape as the knee bends and whether the fat pad provides cushioning in the knee joint. The images were initially processed using a median filter then segmented using a region growing technique to isolate the fat pad from the rest of the knee. Next, rigid registration was performed to align the series of images to match the reference image. Finally, multi-resolution FEM registration was completed between the aligned images. The resulting displacements fields were used to determine the local volume change of the fat pad as the knee bends from extension to flexion through different angles. This multi-angle analysis provides a finer description of the intermediate deformations compared to earlier work, where only a pair of images (full extension and flexion) was analyzed.
\end{abstract}

Keywords: Medical image analysis, local volume change, knee, infrapatellar, Hoffa, segmentation, registration, shape deformation, the Insight Toolkit (ITK)

\section{INTRODUCTION}

The infrapatellar fat pad of Hoffa is located, as shown in Figure 1, inferior to the patella and dorsal to the patellar tendon. The fat pad is covered by synovial membrane and is comprised mainly of structural fat tissue which is not strongly influenced by nutrition. The main purpose of Hoffa's fat pad is thought to provide energy storage in the knee joint however it has been suggested that it may also act as a source of cushioning. ${ }^{1}$ Observing the fat pad using computed tomography (CT) or magnetic resonance imaging (MRI) and studying its structure is important to understanding its appearance and anatomical variations. It can also reveal isolated diseases or pathological conditions of the knee. Abnormalities that are intrinsic to this fat pad include Hoffa disease, intracapsular chondroma, localized nodular synovitis, shear injury, and postarthroscopy and postsurgery fibrosis. ${ }^{2}$ In earlier work the deformation analysis was done on two-angle images, flexion and extension. ${ }^{1}$



Figure 1. A sagittal slice of the infrapatellar (Hoffa) fat pad in relation to the patella, femur and tibia.

${ }^{*}$ Corresponding author: hamarneh@cs.sfu.ca

Medical Imaging 2006: Physiology, Function, and Structure from Medical Images, edited by Armando Manduca, Amir A. Amini, Proc. of SPIE Vol. 6143, 614329, (2006) $\cdot 1605-7422 / 06 / \$ 15 \cdot$ doi: $10.1117 / 12.654301$ 
In order to recognize infrapatellar knee pathology and other potential bio-mechanical function, it is important to not only know the anatomy and anatomical variants, but also understand the shape and appearance variations of the fat pad. For example, variations in the dorsal contour of the infrapatellar fat pad, including indentations, can often be observed. Recent medical image analysis research of the Hoffa fat pad is summarized as follows. Özkur et al studied the prevalence and the shape of a fluid-like indentation, a recess, at the inferior posterior margin of Hoffa's fat pad through MR image examinations of the knee. They examined the location, size and morphology of the recess both visually and using calipers, then performed statistical analysis between patients with and without a recess to correlate this with the incidence of joint effusion. ${ }^{3}$ Kramers-de Quervain et al studied the possible impingement of mobile bearing implants on Hoffa's fat pad in total knee replacement. The impingement can cause knee pain and necessitate early revision surgery. Positron emission tomography (PET) scans of the knee were used to validate fat-pad impingement prior to revision. ${ }^{4}$ Helpert et al presented a pictorial review of tumours and tumour-like lesions of the infrapatellar fat pad of Hoffa with an emphasis on differential diagnosis from MR imaging. ${ }^{5}$ Schweitzer et al used MRI to observe characteristic changes in intracapsular fat pads (including Hoffa's) in patients with proliferative effusions and distinguish them from those with effusions without synovial proliferation. ${ }^{6}$ Another review was also published on Hoffa's fat pad in MRI by Saddik et al. ${ }^{7}$

This paper will investigate the deformation of Hoffa's fat pad at multiple angles of the knee joint - deformation which could potentially provide bio-mechanical functions (such as cushioning) in addition to the classical energy storage role. With the help of a protractor, these angles were determined to be approximately 110, 130, 145, 165 and 180 degrees, and were all taken from the same patient using CT imaging. Although the fat pad itself is routinely visualized and appears clearer on MRI scans of the knee, the fascial boundaries are difficult to identify. Furthermore CT provides better spatial resolution over MRI.

The remainder of this paper is organized as follows: section 2 goes through the processing pipeline which includes the initial imaging, image preprocessing, segmentation, registration, and volume measurements, section 3 contains results of the processed fat pad, and section 4 presents our conclusions.

\section{METHODS}

The following methodologies describe the main components of the image analysis pipeline and are illustrated in Figure 2. The Insight Segmentation and Registration Toolkit $\left(\mathrm{ITK}^{8-13}\right)$ was used for the analysis.

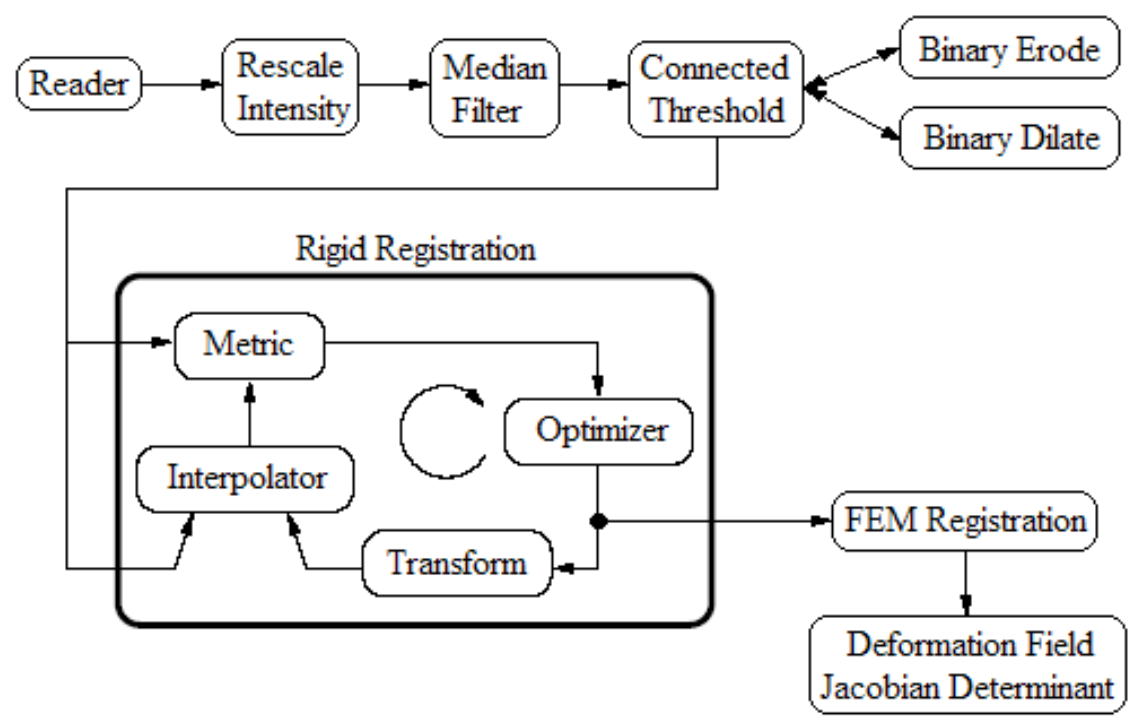

Figure 2. ITK pipeline described in sections $2.2-2.5$ 


\subsection{Imaging}

The following parameters were used during CT imaging for all angles of the knee: $20 \mathrm{~cm}$ FOV, $120 \mathrm{mAs}, 120$ $\mathrm{kV}, 3 \mathrm{x} 1 \mathrm{~mm}$ slice, 0.75 second rotation time, $4 \mathrm{~mm}$ feed/rotation, B45 Kernel. Each 3D image was $512 \mathrm{x} 512 \mathrm{x}$ 126 pixels, capturing a $332 \mathrm{~mm}$ x $332 \mathrm{~mm}$ x $126 \mathrm{~mm}$ physical region of the knee. The image data was stored in DICOM format which was converted into Analyze files using the MRIConvert ${ }^{14}$ software. $^{2}$

\subsection{Image Preprocessing}

A small volume containing primarily the infrapatellar fat pad was manually selected using Volview $2.0^{15}$ to help reduce computation times in ITK during segmentation and registration. The images were then loaded into ITK and fed into a pre-processing pipeline consisting of image intensity rescaling (itk::RescaleIntensityImageFilter) and median filtering (itk::MedianImageFilter). The image intensities were rescaled using the following equation:

$$
\text { outputPixel }=(\text { inputPixel }- \text { inputMin }) \times \frac{(\text { outputMax }- \text { outputMin })}{(\text { inputMax }- \text { inputMin })}+\text { outputMin, }
$$

where inputPixel and outputPixel are the pixel intensities of the input and rescaled images, respectively. The minimum and maximum intensities of the input image are inputMin and inputMax while outputMin and outputMax relate the desired intensity range of the rescaled image. In this case, the images were rescaled from 0 to 255.

\subsection{Image Segmentation}

The pipeline in Figure 3 illustrates the entire segmentation process step by step. First, a connected threshold region growing algorithm (itk::ConnectedThresholdImageFilter) was used to segment the fat pad. Other methods, such as confidence connected and deformable models, were tested to compare their effectiveness in segmentation but neither performed as well as connected threshold. This algorithm required specifying an initial seed location as well as an upper and lower intensity threshold to include during expansion. A series of upper threshold values were tested, with the optimal image containing the fat pad plus some additional layers of skin. Next, a series of operations were performed on the region growing result using itk::BinaryErodeImageFilter and itk::BinaryDilateImageFilter in addition to the connected threshold algorithm described above. The second step was used to isolate the fat pad from the skin layers of similar intensity by eroding the links between them. The third step required another connected threshold operation to reselect just the fat pad while the fourth step finished the opening process by dilating the fat pad back to its original form. The last two steps dilated then eroded the image to effectively close any gaps within the segmented fat pad. All of these functions used a 2-pixel radius structuring element. The image displayed in Figure 4 is an intermediate result after the first connected threshold operation and before the layers of skin were removed.

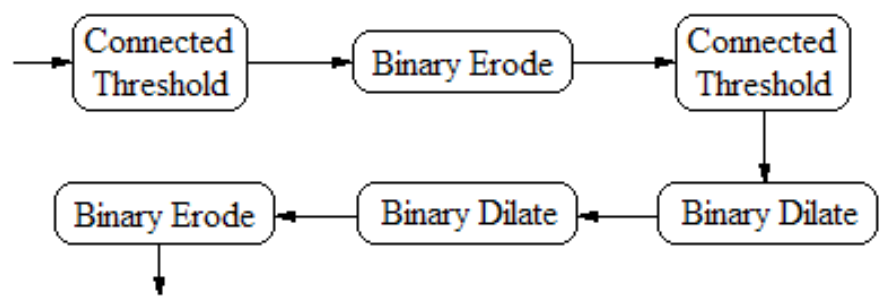

Figure 3. Detailed segmentation pipeline

\subsection{Image Registration}

Two types of registration were required, rigid and non-rigid. The former was used to spatially translate and rotate the images so that they were properly aligned with one another. Note that as the knee bends, the fat pad will rotate; therefore it is important to align the fat pad in each of the images before evaluating the change in 


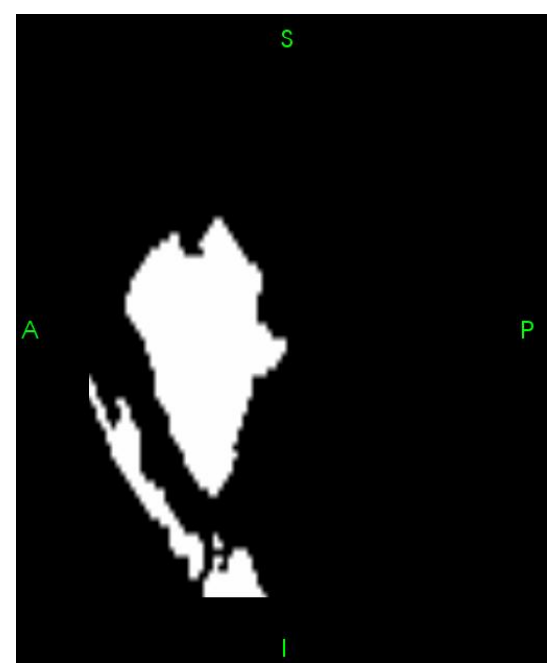

Figure 4. The fat pad has been segmented along with unwanted layers of skin

\begin{tabular}{cc}
\hline Function & Class \\
\hline Similarity Metric & itk::MeanSquaresImageToImageMetric \\
Optimizer & itk::VersorRigid3DTransformOptimizer \\
Transform & itk::VersorRigid3DTransform \\
Interpolator & itk::LinearInterpolateImageFunction \\
\hline
\end{tabular}

Table 1. Classes used for rigid registration.

shape. Table 1 labels the classes used in ITK for rigid registration. Here, the optimizer was set with a maximum step length of 0.2000 , a minimum step length of 0.0001 , and up to 200 iterations. In the latter registration type, a finite element method (FEM) non-rigid registration technique (itk::fem::FEMRegistrationFilter) was used to detect deformations in the pad itself as the knee went from extension to flexion. ${ }^{1,16,17}$ To improve convergence and speed, a multi-resolution FEM registration scheme was used employing 3 levels of increasing resolution.

\subsection{Volume Measurement}

The displacement vectors from the non-rigid registration were used to calculate the local volume changes that deform the Hoffa fat pad from extension to flexion using itk::DeformationFieldJacobianDeterminantFilter. ${ }^{18}$

\section{RESULTS}

The results for the preprocessing analysis are illustrated in Figure 5. These images have been cropped to isolate the fat pad, rescaled to a full intensity range, and median filtered to reduce noise. The images were cropped according to Table 2, where the starting point is the rightmost anterior corner of the cropped 3D image and the size defines its dimensions. Each image has the same dimensions to simplify the segmentation and registration processes.

The images in Figure 6 are a result of the connected threshold method for segmentation. The chosen series of operations, as described in the Methods section, proved to generate the most reliable representations of the Hoffa fat pad. Table 3 specifies the seed point for each cropped image as well as the upper and lower intensity limits to be included during segmentation. Before completion of the opening and closing operations, the threshold filter replaced the intensity value to 250 to contrast the background at 0 .

The rigid transform was the first of two steps in the registration process. Sagittal slices of the resulting spatially translated and rotated images can be seen in Figure 7. In addition, a difference of images was computed between each registered pair of images. This image subtraction is useful as an initial indication of where and 


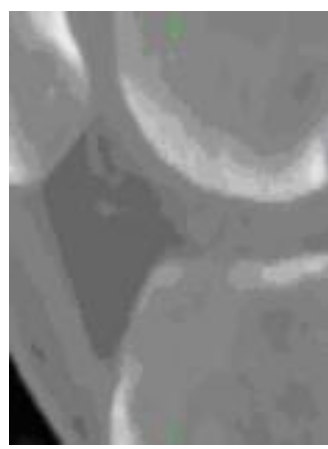

(a)

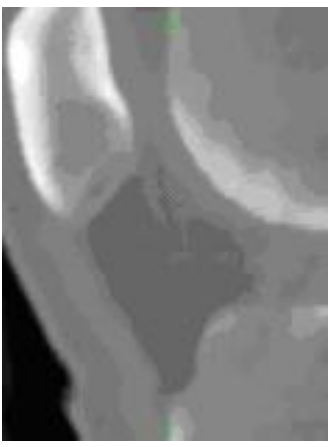

(b)

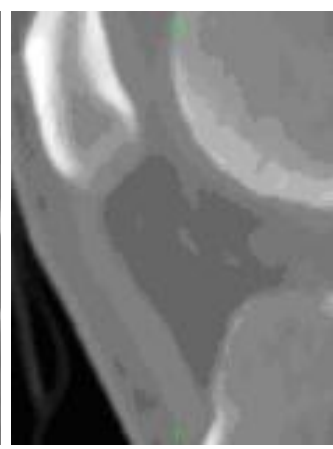

(c)

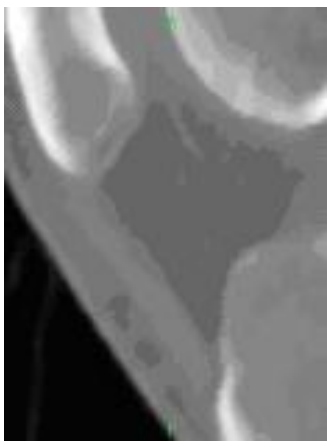

(d)

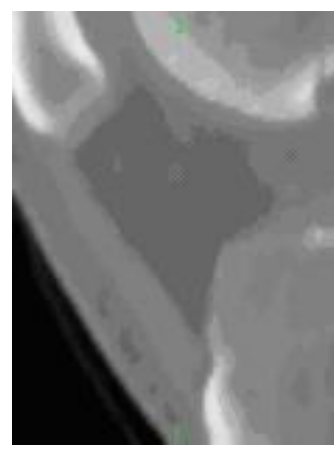

(e)

Figure 5. The preprocessed images are distinguished by the different angles made by the knee. They are (a) 180, (b) 165, (c) 145, (d) 130 and (e) 110 degrees.

\begin{tabular}{ccc}
\hline Joint Angle & Starting Point & Size \\
\hline 180 & $50,260,50$ & $110,80,70$ \\
165 & $80,300,50$ & $110,80,70$ \\
145 & $80,280,40$ & $110,80,70$ \\
130 & $80,300,20$ & $110,80,70$ \\
110 & $90,270,20$ & $110,80,70$ \\
\hline
\end{tabular}

Table 2. Specifications for cropped images in Figure 5.

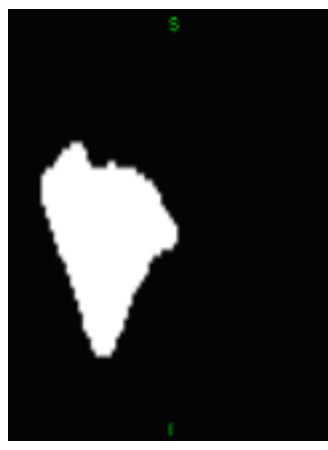

(a)

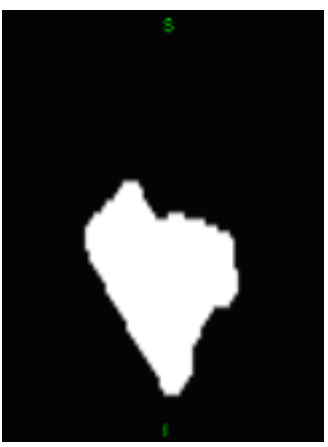

(b)

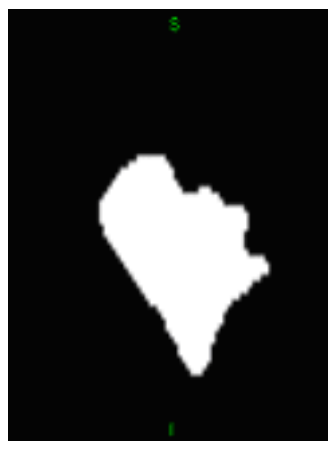

(c)



(d)

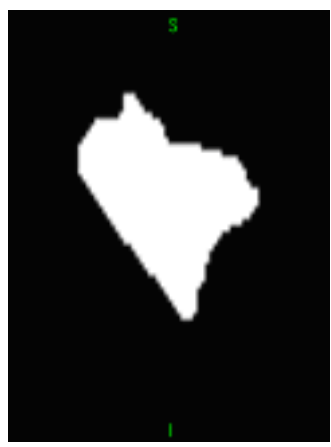

(e)

Figure 6. The Hoffa fat pad has been successfully segmented at angles of (a) 180, (b) 165, (c) 145, (d) 130 and (e) 110 degrees.

by how much the fat pad is compressed. A multi-resolution FEM registration scheme was then used for faster and more robust registration. The resulting displacement vectors fields are displayed in Figure 8 with the help of Amira. ${ }^{19}$ They were produced using the parameters defined in Table 4.

The local changes in volume for a particular sagittal slice are displayed in Figure 9. The contrasts have been adjusted, using MRIcro ${ }^{20}$ to highlight the local volume changes. Relative to each shaded background, the lighter sections indicate expansion while the darker sections indicate contraction.

\section{CONCLUSIONS}

It is important to study the deformation of Hoffa's fat pad in order to better comprehend certain musculoskeletal functions and pathological conditions in the knee. With the recently acquired multi-angle 3D images ranging between flexion and extension, a more accurate and detailed description has been provided of the deformation of the fat. For each imaged angle of the knee, a range of techniques was applied to investigate the fat pad's 


\begin{tabular}{cccc}
\hline Joint Angle & Seed Point & Lower Limit & Upper Limit \\
\hline 180 & $55,55,30$ & 80 & 97 \\
165 & $55,45,30$ & 80 & 97 \\
145 & $55,45,30$ & 80 & 95 \\
130 & $55,37,37$ & 80 & 96 \\
110 & $55,37,37$ & 80 & 96 \\
\hline
\end{tabular}

Table 3. Threshold initial pixel locations and intensity limits.

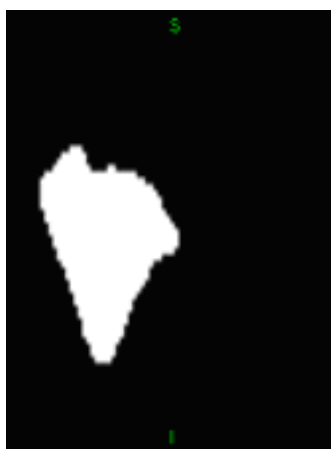

(a)

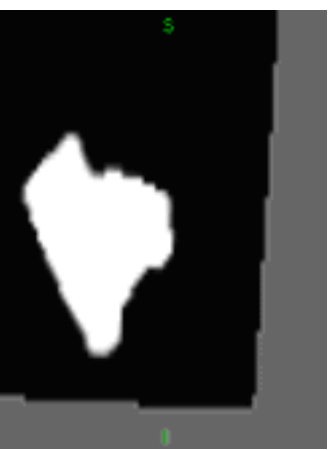

(b)



(c)

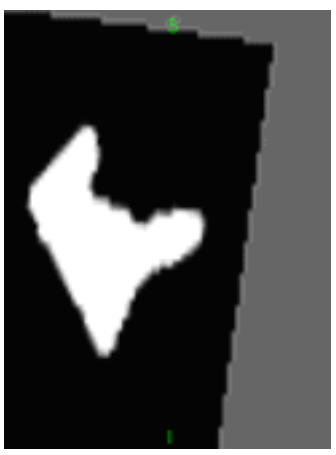

(d)

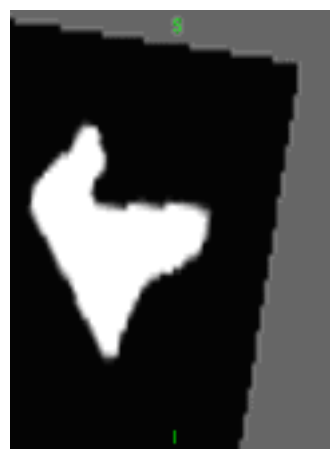

(e)

Figure 7. Images (b) through (e) are the rigid registration results when trying to align with image (a).

morphology and shape deformations. A pipeline was developed in ITK to isolate, segment and register Hoffa's fat pad as well as to calculate the displacement vector field between corresponding images. Each vector field describes the theorized motion of the fat pad modeled as an elastic material using FEM of which the displacements are used to calculate the local volume changes between images. To the best of our knowledge this is the first attempt to analyze the shape deformations of Hoffa's fat pad from CT images of extended and flexed knees at multiple intermediate angles for the purpose of gaining insight into potential mechanical functions of the pad.

\section{ACKNOWLEDGEMENTS}

GH was partly funded by NSERC (Natural Sciences and Engineering Research Council of Canada) discovery grant and Simon Fraser University President's Research Grant Fund. This software implementation relied on the Insight Segmentation and Registration Toolkit (ITK), an open source software developed as an initiative of the U.S. National Library of Medicine (NLM) and available at www.itk.org. The multi-platform configuration tool CMake was used for configuring ITK and facilitating its use from our project. CMake was partially funded by

\begin{tabular}{cc}
\hline Parameters & Values \\
\hline Number of multi-levels & 3 \\
Scaling at lowest level of pyramid & 4 \\
Number of pixels per element & 3 \\
Elasticity & $1.0 \times \mathrm{e} 2$ \\
Density x capacity & $1.0 \times \mathrm{e} 4$ \\
Image energy scaling (gamma) & 1 \\
Number Of integration points & 4 \\
Width Of metric region & 2 \\
Maximum iterations & 2 \\
\hline
\end{tabular}

Table 4. Parameters for multi-resolution FEM registration. 


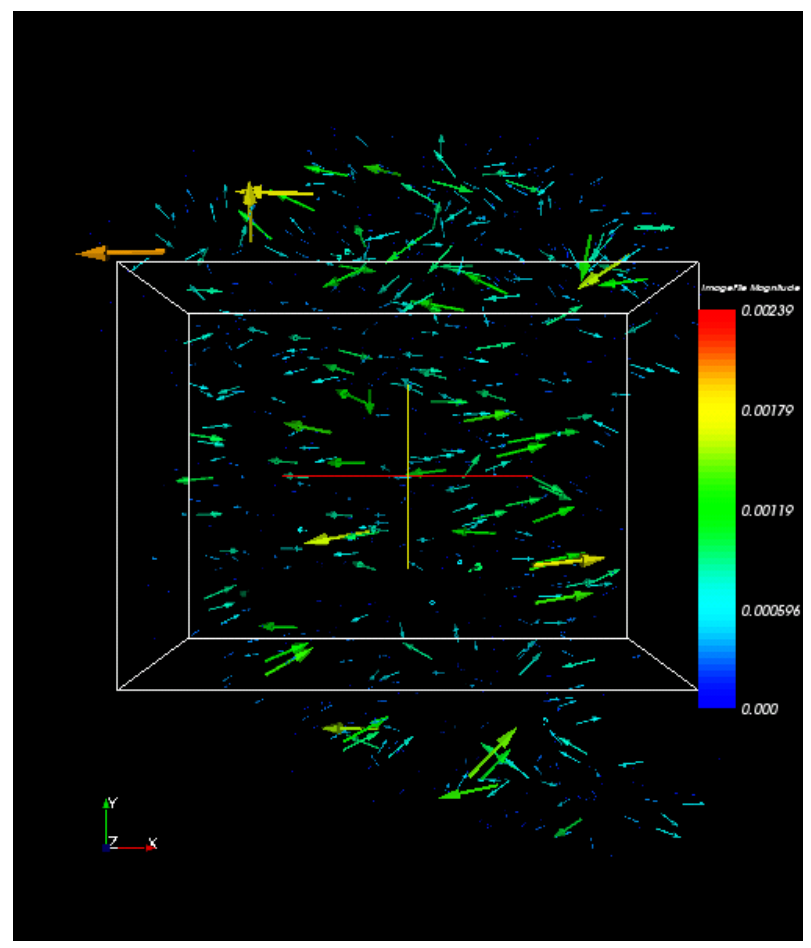

(a)

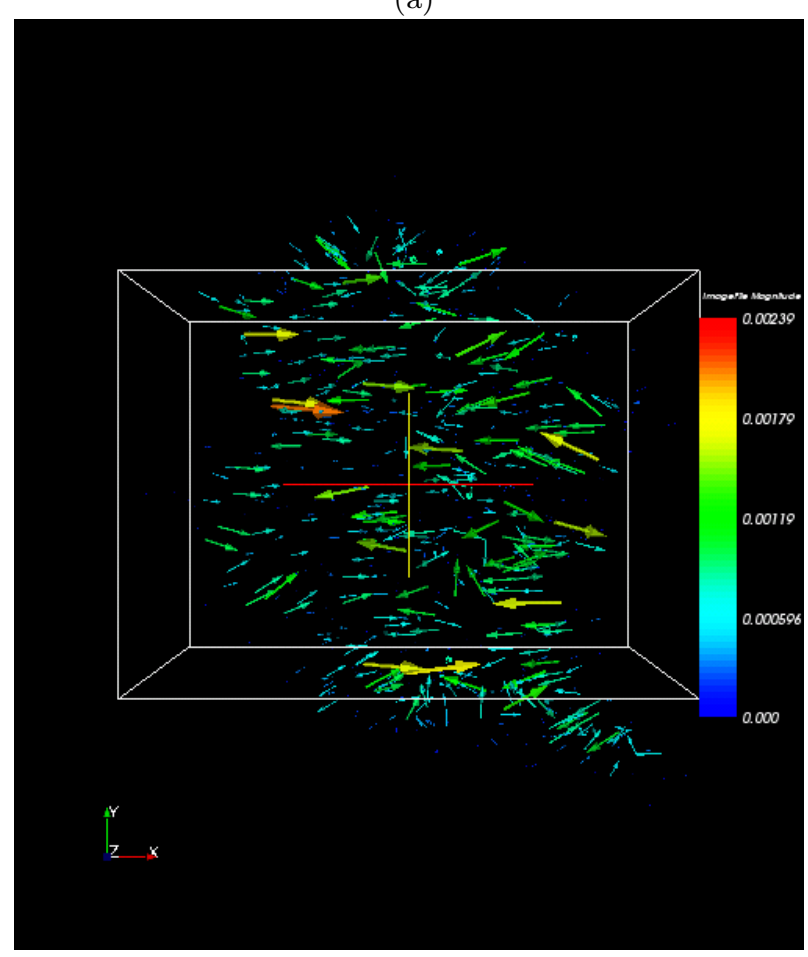

(c)

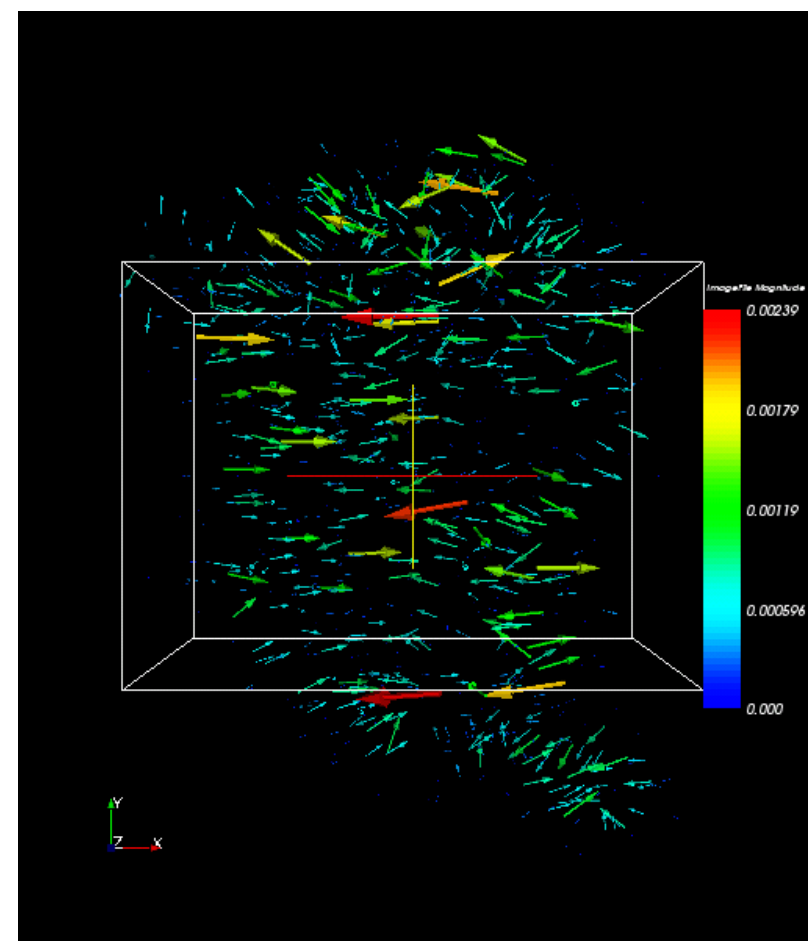

(b)

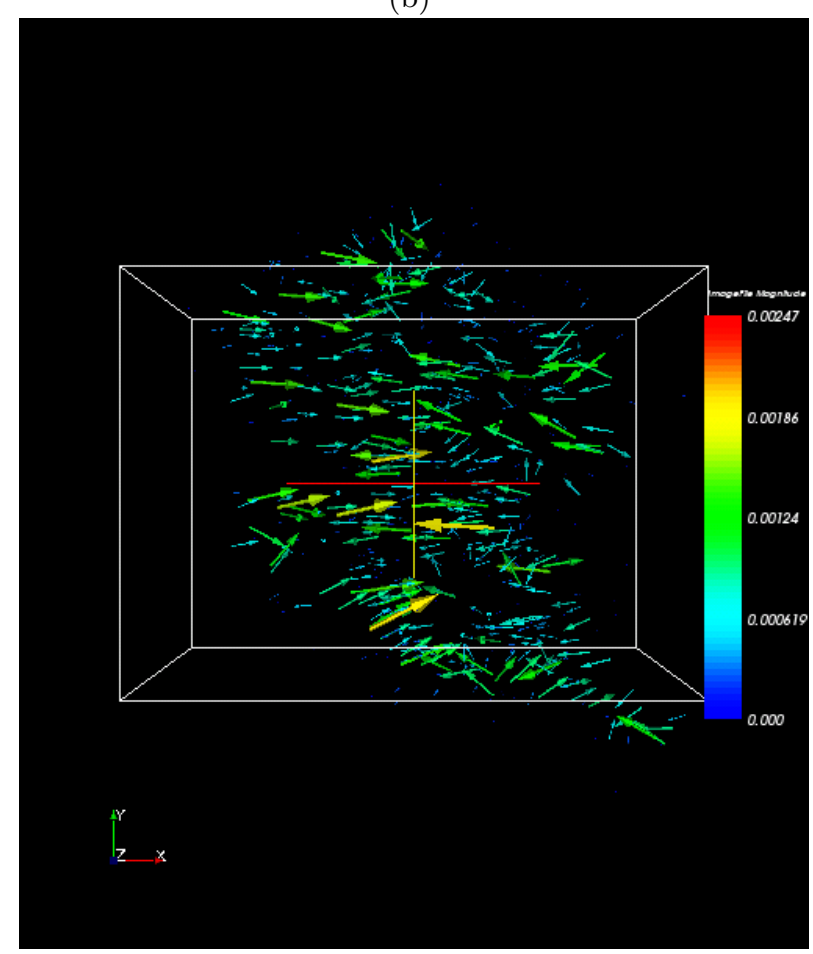

(d)

Figure 8. A three-dimensional view of the resulting displacement vector fields from FEM registration. The arrows increase in magnitude from blue $=0$ to red $=2.5 \mathrm{~mm}$. The joint angles, in degrees, were registered with their adjacent angles as follows: (a) $165 \rightarrow 180$, (b) $145 \rightarrow 165$, (c) $130 \rightarrow 145$, and (d) $110 \rightarrow 130$. 


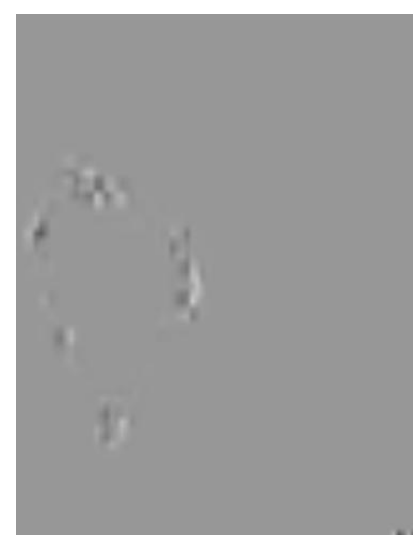

(a)

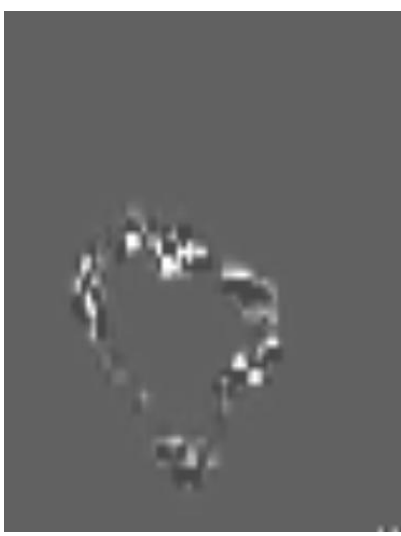

(b)

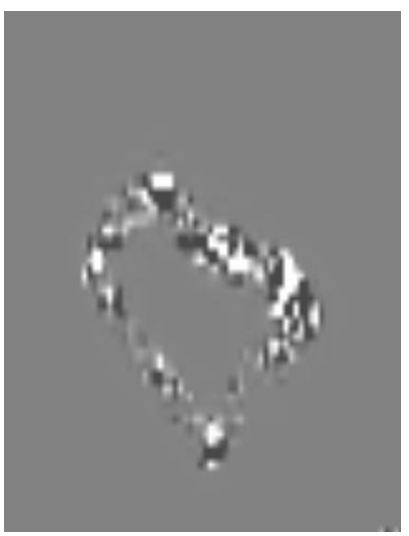

(c)

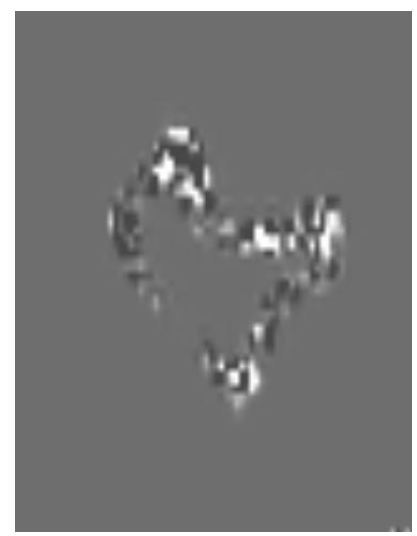

(d)

Figure 9. The local volume changes for a particular slice of the Hoffa fat pad using the displacements vectors from FEM registration. The local changes are as follows: (a) $165 \rightarrow 180$, (b) $145 \rightarrow 165$, (c) $130 \rightarrow 145$, and (d) $110 \rightarrow 130$.

the U.S. National Library of Medicine as part of the Insight Toolkit project. CMake is an open source system and it is freely available at www.cmake.org. The manuscript was improved by comments from Russell Warneboldt.

\section{REFERENCES}

1. G. Hamarneh, V. Chu, M. Bordalo-Rodrigues, and M. Schweitzer, "Deformation analysis of hoffa's fat pad from ct images of knee flexion and extension," Proceedings of SPIE Medical Imaging: Physiology, Function, and Structure from Medical Images 5746, pp. 527-534, 2005.

2. J. Jacobson, L. Lenchik, M. Ruhoy, M. Schweitzer, and D. Resnick, "MR imaging of the infrapatellar fat pad of hoffa.," Radiographics 17(3), pp. 675-691, 1997.

3. A. Ozkur, I. Adaletli, A. Sirikci, R. Kervancioglu, and M. Bayram, "Hoffa's recess in the infrapatellar fat pad of the knee on MR imaging," Surg Radiol Anat. , 2004.

4. I. K.-D. Quervain, I. Engel-Bicik, W. Miehlke, T. Drobny, and U. Munzinger, "Fat-pad impingement after total knee arthroplasty with the LCS A/P-Glide system," Knee Surg Sports Traumatol Arthrosc, 2004.

5. C. Helpert, A. M. Davies, N. Evans1, and R. J. Grimer, "Musculoskeletal differential diagnosis of tumours and tumour-like lesions of the infrapatellar (hoffas) fat pad: pictorial review with an emphasis on MR imaging," in European Radiology, pp. 1432-1084, Springer-Verlag Heidelberg, 2004.

6. M. Schweitzer, A. Falk, M. Pathria, S. Brahme, J. Hodler, and D. Resnick, "MR imaging of the knee: can changes in the intracapsular fat pads be used as a sign of synovial proliferation in the presence of an effusion?," American Journal of Roentgenology 160(4), pp. 823-6, 1993.

7. D. Saddik, E. McNally, and M. Richardson, "MRI of hoffa's fat pad," Skeletal Radiol 33(8), pp. 433-444, 2004.

8. T. Yoo, Insight into Images, A.K. Peters, 2004.

9. L. Ibánẽz, W. Schroeder, L. Ng, and J. Cates, The ITK Software Guide, Kitware Inc., 2003.

10. K. Martin and B. Hoffman, Mastering CMake, A Cross-Platform Build System, Kitware Inc., 2003.

11. T. Yoo, M. J. Ackerman, W. E. Lorensen, W. Schroeder, V. Chalana, S. Aylward, D. Metaxes, and R. Whitaker, "Engineering and algorithm design for an image processing API: A technical report on ITK - the insight toolkit," in In Proc. of Medicine Meets Virtual Reality, J. Westwood, ed., pp. 586-592, IOS Press Amsterdam, 2002.

12. The Insight Segmentation and Registration Toolkit, www.itk.org.

13. CMake: Cross Platform Make, www.cmake.org.

14. MRIConvert, http://lcni.uoregon.edu/downloads/MRIConvert.htm, Lewis Center for Neuroimaging at the University of Oregon.

15. Volview 2.0, http://www.kitware.com/products/volview.html, Kitware. 
16. J. Gee and D. Haynor, "Numerical methods for high dimensional warps," in Chapter in Brain Warping, A. Toga, ed., Academic Press, 1998.

17. J. Gee and R. Bajcsy, "Elastic matching: Continuum mechanical and probabilistic analysis," in Chapter in Brain Warping, A. Toga, ed., Academic Press, 1998.

18. M. K. Chung, K. J. Worsley, T. Paus, C. Cherif, D. L. Collins, J. N. Giedd, J. L. Rapoport, and A. C. Evans, "A unified statistical approach to deformation-based morphometry," NeuroImage, 2001.

19. Amira 3D Visualization Software, http://www.amiravis.com, Mercury Computer Systems.

20. C. Rorden and M. Brett, MRIcro, http://www.sph.sc.edu/comd/rorden/mricro.html. 